\title{
Estimação de valores genéticos para codornas europeias em função dos níveis da relação treonina: lisina da dieta: do nascimento aos 21 dias de idade
}

[Estimation of breeding values for European quails in function of threonine: lysine ratios of diet: from birth to 21 days of age]

\author{
G.Q. Faria ${ }^{1}$, C.M. Bonafé ${ }^{1}$, K.A.R. Souza ${ }^{1}$, M.A. Silva ${ }^{2}$, L.S. Costa ${ }^{1}$, H.J.D. Lima ${ }^{3}$, F.G. Campos ${ }^{1}$, \\ R.B. Silva ${ }^{1}$, A.A. Silva ${ }^{1}$, G. Tarocóo ${ }^{1}$, G.M.F. Rocha ${ }^{1}$, J.A. Miranda ${ }^{1}$ \\ ${ }^{1}$ Universidade Federal dos Vales do Jequitinhonha e Mucuri - Diamantina, MG \\ ${ }^{2}$ Pesquisador Visitante Nacional Sênior - Capes \\ ${ }^{3}$ Universidade Federal de Mato Grosso - Mato Grosso, MT
}

\begin{abstract}
RESUMO
O presente trabalho foi realizado com o objetivo de avaliar a sensibilidade dos valores genéticos dos pesos corporais e as características de carcaças de codornas europeias às mudanças do gradiente ambiental (níveis da relação treonina com a lisina das dietas), do nascimento aos 21 dias de idade, por meio de modelos de regressão aleatória com diferentes classes de variância residual. Os dados utilizados neste estudo são provenientes de 915 codornas de corte da linhagem LF1 e 839 da linhagem LF2, pertencentes ao Programa de Melhoramento Genético da Universidade Federal dos Vales do Jequitinhonha e Mucuri. Foram avaliados os pesos corporais e os rendimentos da carcaça das aves. As sensibilidades dos valores genéticos às mudanças nos níveis da relação treonina:lisina (interação genótipo $\mathrm{x}$ ambiente) foram obtidas por modelos de regressão aleatória (utilizando normas de reação) por meio do programa Wombat, que utiliza o princípio da máxima verossimilhança restrita (REML). O modelo de regressão aleatória que considerou duas classes de variância residual foi o mais indicado para a maioria das análises realizadas. Verificaram-se alterações na classificação dos valores genéticos para as duas linhagens de codornas de corte estudadas. Esse comportamento indica sensibilidade de valores genéticos aditivos às mudanças nutricionais, o que caracteriza a existência de interação genótipo $\mathrm{x}$ ambiente. A predição dos valores genéticos deve ser feita com o mesmo nível da relação treonina:lisina da dieta com a qual as codornas serão alimentadas no sistema de produção.
\end{abstract}

Palavras-chave: Codornas europeias, sensibilidade de valor genético, heterogeneidade de variância residual

\begin{abstract}
This research was carried out to evaluate the sensitivity of breeding values of body weight and carcass traits in two lines of European quails (LF1 and LF2) to changes in the environment gradient (levels of threonine: lysine ratio of diets) from hatch to 21 days of age in two lines LF1 and LF2 using Random Regression Models with different classes of residual variance. Records are from 915 quails of line LF1 and 839 of line LF2 belonging to the Breeding Improvement Program of Universidade Federal dos Vales do Jequitinhonha e Mucuri. Live body weight and weights and yields of carcass, breast, and thigh and drumstick were measured. The sensitivities of breeding values to changes in threonine: lysine ratios (genotype x environment interaction) of diets were obtained by random regression models (reaction model) using the WOMBAT program using the Restricted Maximum Likelihood principle. Model considering two classes of residual variance showed the best goodness of fit. The Reaction Norms analyses indicated changes in the ranking of breeding values for both lines suggesting quails selected in one level of threonine: lysine ratio will not express all their genetic potential if fed different threonine: lysine ratio diets. This behavior indicates sensitivity of breeding values to changes in the nutrition characterizing the genotype by environment interaction. The prediction of breeding values must be performed using the same level of threonine: lysine ratio in diet the quails will be fed in the production system.
\end{abstract}

Keywords: European quails, sensitivity of breeding value, residual variance heterogeneity

Recebido em 18 de novembro de 2015

Aceito em 9 de junho de 2016

E-mail: gabysqueirozfaria@gmail.com 


\section{INTRODUÇÃO}

Várias pesquisas têm buscado informações que possam contribuir para a redução dos custos com a alimentação e dos teores de proteína bruta das dietas, de forma que se minimize o impacto ambiental. A redução no conteúdo de proteína não permite adequado desempenho das aves, sendo necessário observar também as proporções entre os aminoácidos (Felipe et al., 2012).

Os modelos de regressão aleatória (MRA) têm sido propostos como alternativa na avaliação genética de dados longitudinais por admitirem ajustar uma trajetória aleatória para cada indivíduo como desvios de uma trajetória média da população, fazendo, assim, com que cada animal tenha uma forma diferente em seus desempenhos genéticos (Mercadante et al., 2002).

No melhoramento genético animal, o conhecimento da interação genótipo $\mathrm{x}$ ambiente tem se tornado cada vez mais importante. Nessa perspectiva, a presença da interação genótipo $\mathrm{x}$ ambiente pode causar mudança no ordenamento dos valores genéticos dos animais, de modo que aquele que apresenta melhor desempenho em um ambiente pode não apresentar o mesmo potencial em outro ambiente (Felipe et al., 2012).

Dentre as formas de se avaliar a IGA, a norma de reação tem se destacado, sendo ela uma função de covariância que permite atribuir a cada animal dois coeficientes de regressão aleatórios (intercepto e inclinação), que predizem o valor genético em função do gradiente ambiental. Com isso, cada animal terá um valor genético para cada ambiente, caracterizando e considerando a IGA (Rodrigues, 2012).

Observa-se que pesquisas de características de crescimento de codornas de corte ainda são escassas na literatura, havendo necessidade de novos estudos genéticos, para modelo de norma de reação, com o intuito de avaliar a interação genótipo x ambiente, por meio da expressão das características.
Objetivou-se, no presente estudo, avaliar a sensibilidade dos valores genéticos de pesos corporais e as características de carcaças às mudanças nas relações treonina:lisina das dietas de codornas de corte, do nascimento aos 21 dias, usando modelos de regressão aleatória com diferentes classes de variâncias residuais, a fim de buscar maior precisão do número de parâmetros estimados para obtenção dos componentes de (co)variância.

\section{MATERIAL E MÉTODOS}

A pesquisa foi realizada com autorização da Comissão de Ética no Uso de Animais da UFVJM, protocolo número 027/2014.

O experimento foi desenvolvido no Setor de Avicultura, do Departamento de Zootecnia da Universidade Federal dos Vales do Jequitinhonha e Mucuri, localizada em Diamantina-MG.

Foram utilizadas duas linhagens (LF1 e LF2) de codornas de corte (Coturnix coturnix) do Programa de Melhoramento de Aves da UFVJM, sendo o total de1754 aves.

Do nascimento aos 21 dias de idade, as codornas receberam a dieta experimental (os cinco níveis da relação treonina:lisina - Tab. 1); de 22 a 35 dias de idade, foi fornecida dieta única para todos os animais.

As aves foram abatidas aos 35 dias de idade para a avaliação das características de carcaça.

As dietas fornecidas para as aves (Tab. 1) foram balanceadas com base no conteúdo aminoacídico digestível dos alimentos apresentados em Rostagno et al. (2005), e segundo as exigências nutricionais recomendadas por Silva e Costa (2009). A fim de assegurar que nenhum aminoácido ficasse deficiente na dieta, foram acrescentados $3 \%$ nas exigências de cada aminoácido, exceto lisina digestível e treonina digestível. 
Tabela 1. Composição e valor nutricional das dietas experimentais, na matéria natural, para codornas de corte de 1 a 21 dias de idade

\begin{tabular}{|c|c|c|c|c|c|}
\hline \multirow{2}{*}{ Ingredientes $(\mathrm{g} / \mathrm{kg})$} & \multicolumn{5}{|c|}{ Relação treonina:lisina } \\
\hline & 0,66 & 0,71 & 0,76 & 0,81 & 0,86 \\
\hline & \multicolumn{5}{|c|}{ Composição (\%) } \\
\hline Milho moído & 48,96 & 48,96 & 48,96 & 48,96 & 48,96 \\
\hline Farelo de soja (45\%) & 44,82 & 44,82 & 44,82 & 44,82 & 44,82 \\
\hline Óleo de soja & 1,61 & 1,61 & 1,61 & 1,61 & 1,61 \\
\hline Calcário & 1,20 & 1,20 & 1,20 & 1,20 & 1,20 \\
\hline Fosfato bicálcico & 1,10 & 1,10 & 1,10 & 1,10 & 1,10 \\
\hline Sal comum & 0,38 & 0,38 & 0,38 & 0,38 & 0,38 \\
\hline Mistura mineral $^{1}$ & 0,20 & 0,20 & 0,20 & 0,20 & 0,20 \\
\hline Mistura vitamínica $^{2}$ & 0,20 & 0,20 & 0,20 & 0,20 & 0,20 \\
\hline Cloreto de colina ( $60 \%)$ & 0,01 & 0,01 & 0,01 & 0,01 & 0,01 \\
\hline Antioxidante $^{3}$ & 0,01 & 0,01 & 0,01 & 0,01 & 0,01 \\
\hline Coccidiostático $^{4}$ & 0,05 & 0,05 & 0,05 & 0,05 & 0,05 \\
\hline DL- metionina & 0,37 & 0,37 & 0,37 & 0,37 & 0,37 \\
\hline L- treonina & 0,00 & 0,05 & 0,12 & 0,19 & 0,25 \\
\hline L- isoleucina & 0,09 & 0,09 & 0,09 & 0,09 & 0,09 \\
\hline L- arginina & 0,20 & 0,20 & 0,20 & 0,20 & 0,20 \\
\hline L-glutâmico & 0,30 & 0,25 & 0,18 & 0,11 & 0,05 \\
\hline Amido & 0,50 & 0,50 & 0,50 & 0,50 & 0,50 \\
\hline Total & 100,00 & 100,00 & 100,00 & 100,00 & 100,00 \\
\hline Proteína bruta (\%) & 24,54 & 24,54 & 24,54 & 24,54 & 24,54 \\
\hline Cálcio $(\%)$ & 0,85 & 0,85 & 0,85 & 0,85 & 0,85 \\
\hline Fósforo disponível (\%) & 0,32 & 0,32 & 0,32 & 0,32 & 0,32 \\
\hline Sódio $(\%)$ & 0,17 & 0,17 & 0,17 & 0,17 & 0,17 \\
\hline Fibra bruta (\%) & 3,27 & 3,27 & 3,27 & 3,27 & 3,27 \\
\hline \multicolumn{6}{|l|}{ Aminoácidos digestíveis (\%) } \\
\hline Lisina & 1,25 & 1,25 & 1,25 & 1,25 & 1,25 \\
\hline Metionina + cistina & 1,02 & 1,02 & 1,02 & 1,02 & 1,02 \\
\hline Triptofano & 0,28 & 0,28 & 0,28 & 0,28 & 0,28 \\
\hline Treonina & 0,84 & 0,88 & 0,95 & 1,02 & 1,07 \\
\hline Arginina & 1,80 & 1,80 & 1,80 & 1,80 & 1,80 \\
\hline Isoleucina & 1,07 & 1,07 & 1,07 & 1,07 & 1,07 \\
\hline Valina & 1,04 & 1,04 & 1,04 & 1,04 & 1,04 \\
\hline
\end{tabular}

${ }^{1}$ Composição/kg de produto: manganês: $160 \mathrm{~g}$, ferro: $100 \mathrm{~g}$, zinco: $100 \mathrm{~g}$, cobre: $20 \mathrm{~g}$, cobalto: $2 \mathrm{~g}$, iodo: $2 \mathrm{~g}$. Excipiente q.s.p.: $1000 \mathrm{~g}$.

${ }^{2}$ Composição/kg de produto: vit. A: $12.000 .000 U$., vit $\mathrm{D}_{3}$ : $3.600 .000 \mathrm{UI}$, vit. E: $3.500 \mathrm{UI}$, vit $\mathrm{B}_{1}: 2.500 \mathrm{mg}$, vit $\mathrm{B}_{2}$ : $8.000 \mathrm{mg}$, vit $\mathrm{B}_{6}: 5.000 \mathrm{mg}$, ácido pantotênico: $12.000 \mathrm{mg}$, biotina: $200 \mathrm{mg}$, vit. K: $3.000 \mathrm{mg}$, ácido fólico: $1.500 \mathrm{mg}$, ácido nicotínico: $40.000 \mathrm{mg}$, vit. $\mathrm{B}_{12}: 20.000 \mathrm{mg}$, selênio: $150 \mathrm{mg}$. Veículo q.s.p.: $1.000 \mathrm{~g}$.

${ }^{3}$ Butil-hidróxitolueno. ${ }^{4}$ Salinomicina $12 \%$.

Dos 22 aos 35 dias de idade, todas as codornas foram alimentadas à vontade, com dieta contendo $26,12 \%$ de proteína bruta e $2900 \mathrm{kcal}$ $\mathrm{EM} / \mathrm{kg}$.

$\mathrm{Na}$ modelagem da variância residual, foram consideradas classes de acordo com os níveis de treonina na dieta, sendo que para a variância residual homogênea: CL1 (uma classe) foi considerado o intervalo total de 0,66 a 0,86 de treonina na dieta. Para as variâncias residuais heterogêneas, foram considerados os intervalos: CL2 (duas classes): 0,66 a 0,70 e 0,71 a 0,86 ; CL3 (três classes): 0,66 a 0,70, 0,71 a 0,75 e 0,76 a 0,86; CL4 (quatro classes): 0,66 a 0,70, 0,71 a $0,75,0,76$ a 0,80 e 0,81 a 0,86 , com o total de quatro diferentes modelos para serem utilizados nas análises dos dados. 
O modelo de norma de reação para análise dos dados é representado por:

$$
y_{k i j}=\sum_{k=0}^{1} b_{j k} x_{k}(i)+\sum_{k=0}^{1} a_{k i k} x_{k}(i)+e_{k i j}
$$

em que: $y_{h i j}$ é a observação da codorna $h$, do sexo $j$, alimentada com dieta de nível da relação treonina:lisina $i ; b_{j k}$ é o coeficiente de regressão fixo $k$ atribuído ao sexo $j ; a_{h k}$ é o coeficiente de regressão aleatória $k$ de efeito genético aditivo direto atribuído à codorna $h ; x_{k}(i)$ é o polinômio de Legendre $k$ associado ao nível da relação treonina:lisina $i$; e $e_{h i j}$ é o resíduo associado à observação $y_{h i j}$, sendo este o único efeito que não foi considerado uma função da relação de treonina:lisina das dietas.

O modelo em notação matricial é descrito como:

$$
y=X b+Z a+e,
$$

em que: y é o vetor de observações; $b$ é o vetor com coeficientes de regressão relativos aos efeitos fixos de sexo e da trajetória média de crescimento; $a$ é o vetor com coeficientes de regressão aleatória atribuídos ao efeito genético aditivo direto; $X$ e $Z$ são matrizes de incidência dos efeitos em $b$ e $a$; e $e$ é o vetor dos resíduos. Ambas as trajetórias média e aleatória de crescimento foram modeladas por polinômio de Legendre de segunda ordem relativo ao nível da relação treonina:lisina utilizada nas dietas.

Para avaliação do número de classes de variância residual, foi considerado o critério de informação bayesiano (BIC - Bayesian Information Criterion), proposto por Schwarz (1978).

$$
B I C=-2 \log L+p \log (n),
$$

em que: $L$ refere-se ao valor maximizado da função de verossimilhança do modelo ajustado; $p$ é o número de parâmetros livres a serem estimados; $n$ é o número de observações ou tamanho da amostra; e Log L, o logaritmo da função de máxima verossimilhança restrita (Wolfinger, 1993). Menores valores de BIC indicam modelos mais adequados (Burnham e Anderson, 2004).
Considera-se ainda que:

$$
\begin{aligned}
& E(y)=X B \\
& G=\left[\begin{array}{cc}
\sigma_{b_{0}}^{2} & \sigma_{\partial_{0} \dot{o}_{1}} \\
\sigma_{b_{0} \partial_{1}} & \sigma_{\partial_{1}}^{2}
\end{array}\right] \cdot q \\
& V(y)=Z(A \otimes G) Z^{r}+I_{n} \sigma_{\theta q}^{2} \\
& V\left[\begin{array}{l}
a \\
e
\end{array}\right]=\left[\begin{array}{cc}
A \otimes & \phi \\
\phi & I_{n} \sigma_{\varepsilon}^{2}
\end{array}\right]_{, \emptyset}
\end{aligned}
$$

em que: $\bar{\sigma}_{b_{0}}^{2}$ e $\sigma_{b_{1}}^{2}$ são componentes de variância atribuídos aos coeficientes de regressão constante e linear do efeito genético aditivo direto; $\sigma_{b_{0} b_{1}}$ é o componente de covariância entre os coeficientes de regressão aleatórios do intercepto e linear do efeito genético aditivo direto; $\sigma_{\theta}^{2}$ é o componente de variância residual; $A$ é a matriz de numeradores dos coeficientes de parentesco de Wright; $n$ é o número de observações; $G$ é a matriz de covariância genética aditiva; $\phi$ é o polinômio de Legendre; e $I_{n}$ é a matriz identidade.

Após a obtenção de $G$, estimações das variâncias e covariâncias genéticas aditivas diretas, para qualquer nível de treonina ou combinação de níveis de treonina, tornaram-se possíveis e foram obtidas por meio de funções de covariância (Kirkpatrick et al., 1990). A partir dessas funções, obtém-se a estrutura de covariância genética aditiva direta em função do nível de treonina da dieta. Assim, a covariância genética aditiva direta entre os níveis de treonina $i$ e $j$,

$\sigma_{a i j}$ foi obtida por:

$$
\sigma_{a i j}=Z_{i} G Z_{j, \emptyset}^{r},
$$

em que:

$$
\begin{gathered}
Z_{i}=\left[\begin{array}{ll}
x_{0}(i) & x_{1}(i)
\end{array}\right] \\
Z_{j}=\left[\begin{array}{ll}
x_{0}(j) & x_{1}(j)
\end{array}\right] .
\end{gathered}
$$

A estimativa da herdabilidade direta em função do nível de treonina da dieta e da classe residual foi feita da seguinte maneira:

$$
h_{d_{i}}^{2}=\frac{Z_{i} G Z_{i}^{\prime}}{Z_{i} G Z_{i}^{r}+\sigma_{e_{j}}^{2}}
$$


em que: $i$ representa o nível de treonina da dieta; j refere-se à classe residual da qual este nível faz parte; e $h_{d_{\hat{f}}}^{2}$ é a herdabilidade direta para o nível de treonina:lisina $\mathrm{i}$.

Os componentes de covariância atribuídos a cada efeito aleatório foram estimados por meio do programa Meyer (2006), que utiliza o método da máxima verossimilhança restrita (REML). Funções de covariância foram utilizadas para estimar a estrutura de covariância genética aditiva, a herdabilidade direta em função dos níveis de treonina da dieta e as classes de variâncias residuais. $\mathrm{O}$ critério de convergência adotado foi $1 \times 10^{-11}$.

\section{RESULTADOS E DISCUSSÃO}

Houve efeito da interação entre a relação treonina:lisina para as características estudadas, sugerindo que o melhor desempenho foi estimado para codornas alimentadas com o maior nível da relação treonina:lisina, ou seja, 0,86.

Para a avaliação da interação genótipo $\mathrm{x}$ ambiente, o modelo que considerou duas classes de heterogeneidade de variância residual (Tab. 2 e 3) foi mais indicado, exceto para peso aos 14 dias, rendimento da carcaça e rendimento da coxa + sobrecoxa na linhagem LF1. Portanto, adotou-se o modelo com duas classes de variância residual para todas as análises. Menores valores de BIC indicam modelos mais adequados (Burnham e Anderson, 2004).

Tabela 2. Melhores modelos que incluem variância residual pelo critério de informação baysiano (BIC)

\begin{tabular}{ccccc}
\hline \multirow{2}{*}{ Linhagem } & Peso corporal $^{1}$ & $\begin{array}{c}\text { Classes de } \\
\text { variância residual }\end{array}$ & $\begin{array}{c}\text { Número de } \\
\text { parâmetros }\end{array}$ & BIC $^{2}$ \\
\hline \multirow{3}{*}{ LF1 } & P14 & 4 & 7 & $1.957,149$ \\
& P21 & 2 & 5 & $2.289,859$ \\
& P28 & 2 & 5 & $2.482,929$ \\
P35 & 2 & 5 & $2.697,772$ \\
\hline \multirow{3}{*}{ LF2 } & P14 & 2 & 5 & $1.680,013$ \\
& P21 & 2 & 5 & $2.045,733$ \\
& P28 & 2 & 5 & $2.192,179$ \\
\hline
\end{tabular}

${ }^{1} \mathrm{P} 14, \mathrm{P} 21, \mathrm{P} 28$ e $\mathrm{P} 35=$ pesos corporais aos 14, 21, 28 e 35 dias de idade, respectivamente.

${ }^{2}$ Critério de informação baysiano.

Tabela 3. Melhores modelos que incluem variância residual pelo critério de informação baysiano (BIC)

\begin{tabular}{ccccc}
\hline Linhagem & Característica $^{1}$ & $\begin{array}{c}\text { Classes de variância } \\
\text { residual }\end{array}$ & $\begin{array}{c}\text { Número de } \\
\text { parâmetros }\end{array}$ & BIC $^{2}$ \\
\hline \multirow{4}{*}{ LF1 } & PPA & 2 & 5 & 2479,303 \\
& Pcarc & 2 & 5 & 2377,545 \\
& Rcarc & 4 & 7 & 980,304 \\
& Ppeito & 2 & 5 & 1921,157 \\
& Rpeito & 2 & 5 & 877,643 \\
Pcsc & 2 & 5 & 1433,269 \\
Rcsc & 4 & 7 & 569,6901 \\
\hline LF2 & PPA & 2 & 5 & 2173,145 \\
& Pcarc & 2 & 5 & 2051,045 \\
& Rcarc & 2 & 5 & 890,704 \\
& Ppeito & 2 & 5 & 1649,269 \\
Rpeito & 2 & 5 & 748,2018 \\
& Pcsc & 2 & 5 & 1227,686 \\
Rcsc & 2 & 5 & 506,299 \\
\hline
\end{tabular}

${ }^{1} \mathrm{PPA}=$ peso pré-abate, Pcarc $=$ peso da carcaça, Rcarc $=$ rendimento da carcaça, Ppeito $=$ peso do peito, Rpeito $=$ rendimento do peito, $\mathrm{Pcsc}=$ peso da coxa + sobrecoxa e Rcsc $=$ rendimento da coxa + sobrecoxa.

${ }^{2}$ Critério de informação baysiano. 
Para peso aos 14 dias (LF1 - acima da diagonal principal) e para peso aos 21 dias (LF2 - abaixo da diagonal principal), com base nas recomendações de Robertson (1959), (correlações menores que 0,80 ), observa-se que ocorre interação genótipo x ambiente (Tab. 4) entre os níveis 0,66 e 0,86 da relação treonina:lisina.

Para peso aos 28 e 35 dias de idade em ambas as linhagens, peso aos 14 dias (LF2) e peso aos 21 dias (LF1), as estimativas de correlações genéticas foram todas maiores que 0,80, indicando que a maioria dos genes que atua em um nível de treonina atua nos demais (Robertson, 1959).

Para a linhagem LF2, a correlação genética entre os níveis da relação treonina:lisina 0,66 e 0,86 para peso pré-abate é menor que 0,8 , indicando interação genótipo $\mathrm{x}$ ambiente branda. Para $\mathrm{o}$ peso da coxa + sobrecoxa, ocorre interação entre os níveis $(0,66$ e 0,86$)$, nos quais os valores de correlação genética estimados foram menores que 0,80 , e para rendimento da coxa + sobrecoxa, observam-se valores menores que 0,8 na interação entre o nível 0,66 e os demais níveis da relação treonina:lisina (Tab. 4).

Tabela 4. Estimativas das correlações genéticas entre os pesos corporais e os rendimentos de carcaça nos diferentes níveis da relação treonina:lisina para as linhagens LF1 (acima da diagonal) e LF2 (abaixo da diagonal)

\begin{tabular}{|c|c|c|c|c|c|c|c|c|c|c|c|}
\hline \multicolumn{6}{|c|}{ Peso corporal aos 14 dias de idade } & \multicolumn{6}{|c|}{ Peso da carcaça } \\
\hline $\begin{array}{l}\text { Níveis da relação } \\
\text { treonina:lisina }\end{array}$ & 0,66 & 0,71 & 0,76 & 0,81 & 0,86 & $\begin{array}{l}\text { Níveis da relação } \\
\text { treonina:lisina }\end{array}$ & 0,66 & 0,71 & 0,76 & 0,81 & 0,86 \\
\hline 0,66 & & 0,987 & 0,940 & 0,848 & 0,715 & 0,66 & & 1 & 0,998 & 0,995 & 0,992 \\
\hline 0,71 & 0,985 & & 0,982 & 0,921 & 0,817 & 0,71 & 0,998 & - & 0,999 & 0,998 & 0,995 \\
\hline 0,76 & 0,935 & 0,982 & & 0,978 & 0,911 & 0,76 & 0,989 & 0,997 & - & 0,999 & 0,998 \\
\hline 0,81 & 0,851 & 0,929 & 0,982 & & 0,977 & 0,81 & 0,968 & 0,982 & 0,994 & & 0,999 \\
\hline 0,86 & 0,744 & 0,849 & 0,933 & 0,984 & - & 0,86 & 0,921 & 0,944 & 0,968 & 0,989 & - \\
\hline \multicolumn{6}{|c|}{ Peso corporal aos 21 dias de idade } & \multicolumn{6}{|c|}{ Rendimento da carcaça } \\
\hline $\begin{array}{c}\text { Níveis da relação } \\
\text { treonina:lisina }\end{array}$ & 0,66 & 0,71 & 0,76 & 0,81 & 0,86 & $\begin{array}{l}\text { Níveis da relação } \\
\text { treonina:lisina }\end{array}$ & 0,66 & 0,71 & 0,76 & 0,81 & 0,86 \\
\hline 0,66 & & 1 & 0,998 & 0,995 & 0,991 & 0,66 & & 0,999 & 0,997 & 0,987 & 0,958 \\
\hline 0,71 & 0,985 & & 0,999 & 0,998 & 0,994 & 0,71 & 0,986 & - & 0,999 & 0,992 & 0,967 \\
\hline 0,76 & 0,937 & 0,983 & & 0,999 & 0,997 & 0,76 & 0,956 & 0,991 & 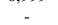 & 0,997 & 0,979 \\
\hline 0,81 & 0,856 & 0,932 & 0,983 & - & 0,999 & 0,81 & 0,920 & 0,972 & 0,995 & - & 0,991 \\
\hline 0,86 & 0,752 & 0,854 & 0,935 & 0,985 & - & 0,86 & 0,884 & 0,949 & 0,983 & 0,997 & 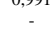 \\
\hline \multicolumn{6}{|c|}{ Peso corporal aos 28 dias de idade } & \multicolumn{6}{|c|}{ Peso do peito } \\
\hline $\begin{array}{l}\text { Níveis da relação } \\
\text { treonina:lisina }\end{array}$ & 0,66 & 0,71 & 0,76 & 0,81 & 0,86 & $\begin{array}{l}\text { Níveis da relação } \\
\text { treonina:lisina }\end{array}$ & 0,66 & 0,71 & 0,76 & 0,81 & 0,86 \\
\hline 0,66 & - & 0,999 & 0,996 & 0,990 & 0,981 & 0,66 & - & 0,988 & 0,937 & 0,836 & 0,667 \\
\hline 0,71 & 0,999 & & 0,999 & 0,995 & 0,988 & 0,71 & 0,999 & -0 & 1 & 0,908 & 0,776 \\
\hline 0,76 & 0,996 & 0.999 & - & 0,999 & 0,994 & 0,76 & 0,997 & 0,999 & - & 0,973 & 0,885 \\
\hline 0,81 & 0,991 & 0,996 & 0,999 & - & 0,998 & 0,81 & 0,993 & 0,997 & 0,997 & & 0,969 \\
\hline 0,86 & 0,985 & 0,992 & 0,996 & 0,999 & - & 0,86 & 0,988 & 0,993 & 0,996 & 0,999 & - \\
\hline \multicolumn{6}{|c|}{ Peso corporal aos 35 dias de idade } & \multicolumn{6}{|c|}{$\begin{array}{l}\text { 0,900 } 0,99 \\
\text { Rendimento do peito } \\
\end{array}$} \\
\hline $\begin{array}{c}\text { Níveis da relação } \\
\text { treonina:lisina }\end{array}$ & 0,66 & 0,71 & 0,76 & 0,81 & 0,86 & $\begin{array}{c}\text { Níveis da relação } \\
\text { treonina:lisina }\end{array}$ & 0,66 & 0,71 & 0,76 & 0,81 & 0,86 \\
\hline 0,66 & & 1 & 0,998 & 0,995 & 0,991 & 0,66 & & 0,997 & 0,988 & 0,973 & 0,952 \\
\hline 0,71 & 0,998 & & 0,999 & 0,998 & 0,995 & $\begin{array}{l}0,00 \\
0,71\end{array}$ & 0,984 & - & 0,997 & 0,988 & 0,973 \\
\hline 0,76 & 0,994 & 0,999 & 0,999 & 0,999 & 0,998 & 0,76 & 0,958 & 0,994 & 0,991 & 0,997 & 0,988 \\
\hline 0,81 & 0,987 & 0,995 & 0,999 & & 0,999 & 0,81 & 0,936 & 0,984 & 0,997 & & 0,997 \\
\hline $\begin{array}{l}0,01 \\
0,86\end{array}$ & 0,979 & 0,989 & 0,996 & 0,999 & , & $\begin{array}{l}0,81 \\
0,86\end{array}$ & $\begin{array}{l}0,930 \\
0,917\end{array}$ & 0,974 & 0,993 & 0,999 & - \\
\hline \multicolumn{6}{|c|}{ Peso pré-abate } & \multicolumn{6}{|c|}{ Peso de coxa + sobrecoxa } \\
\hline $\begin{array}{c}\text { Níveis da relação } \\
\text { treonina:lisina }\end{array}$ & 0,66 & 0,71 & 0,76 & 0,81 & 0,86 & $\begin{array}{c}\text { Níveis da relação } \\
\text { treonina:lisina }\end{array}$ & 0,66 & 0,71 & 0,76 & 0,81 & 0,86 \\
\hline 0,66 & - & 1 & 0,998 & 0.996 & 0,993 & 0,66 & - & 1 & 0,998 & 0,994 & 0,987 \\
\hline 0,71 & 0,990 & & 1 & 0,998 & 0,996 & 0,71 & 0,975 & - & 0,999 & 0,997 & 0,991 \\
\hline 0,76 & 0,951 & 0,985 & - & 1 & 0,998 & 0,76 & 0,905 & 0,977 & 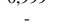 & 0,999 & 0,995 \\
\hline & 0,870 & 0,833 & 0,98 & - & 1 & 0,81 & 0,808 & 0.918 & 0,982 & - & 0,998 \\
\hline \multirow[t]{8}{*}{0,86} & 0,746 & 0,833 & 0,916 & 0,977 & 1 & $\begin{array}{l}0,81 \\
0,86\end{array}$ & 0,703 & 0,843 & 0,938 & 0,987 & 0,998 \\
\hline & & & & & & \multicolumn{5}{|c|}{ Rendimento de coxa + sobrecoxa } & \\
\hline & & & & & & $\begin{array}{l}\text { Níveis da relação } \\
\text { treonina:lisina }\end{array}$ & 0,66 & 0,71 & 0,76 & 0,81 & 0,86 \\
\hline & & & & & & 0,66 & & 0,760 & 0,451 & 0,273 & 0,170 \\
\hline & & & & & & 0,71 & 0,998 & . & 0,923 & 0,833 & 0,770 \\
\hline & & & & & & 0,76 & 0,992 & 0,998 & - & 0,982 & 0,956 \\
\hline & & & & & & 0,81 & 0,985 & 0,995 & 0,999 & & 0,994 \\
\hline & & & & & & 0,86 & 0,978 & 0,990 & 0,997 & 0,999 & - \\
\hline
\end{tabular}

Para as duas linhagens (LF1 e LF2), ocorrem interação genótipo $\mathrm{x}$ ambiente para as características de peso e rendimento da carcaça e peso e rendimento do peito. As correlações foram altas, maiores que 0,8 , assim como para a linhagem LF1 para as características de peso da coxa + sobrecoxa e peso pré-abate.
A correlação entre os coeficientes de regressão aleatória, intercepto $\left(b_{0}\right)$ e linear $\left(b_{1}\right)$, da LF1 foi positiva para rendimento do peito e rendimento da coxa + sobrecoxa; para a linhagem LF2, houve correlação positiva (entre $b_{0}$ e $b_{1}$ ) para peso aos 28 dias, peso aos 35 dias, rendimento da carcaça, rendimento do 
peito, peso e rendimento da coxa + sobrecoxa (Tab. 5). Todavia, para a linhagem LF1, a correlação foi negativa para peso aos 14, 21, 28, 35 dias de idade, peso pré-abate, peso do peito, peso e rendimento da carcaça e peso de coxa + sobrecoxa. Quanto maior for a diferença da estimativa da correlação entre $b_{0}$ e $b_{1}$ em relação ao valor 1 , maior será a possibilidade de reordenamento de valores genéticos nos diferentes ambientes (Su et al., 2006).

Tabela 5. Componentes de variância dos coeficientes de regressão aleatórios (b0 e b1) do efeito genético aditivo direto em função dos níveis da relação treonina:lisina da dieta e variâncias residuais, estimados para as linhagens LF1 e LF2

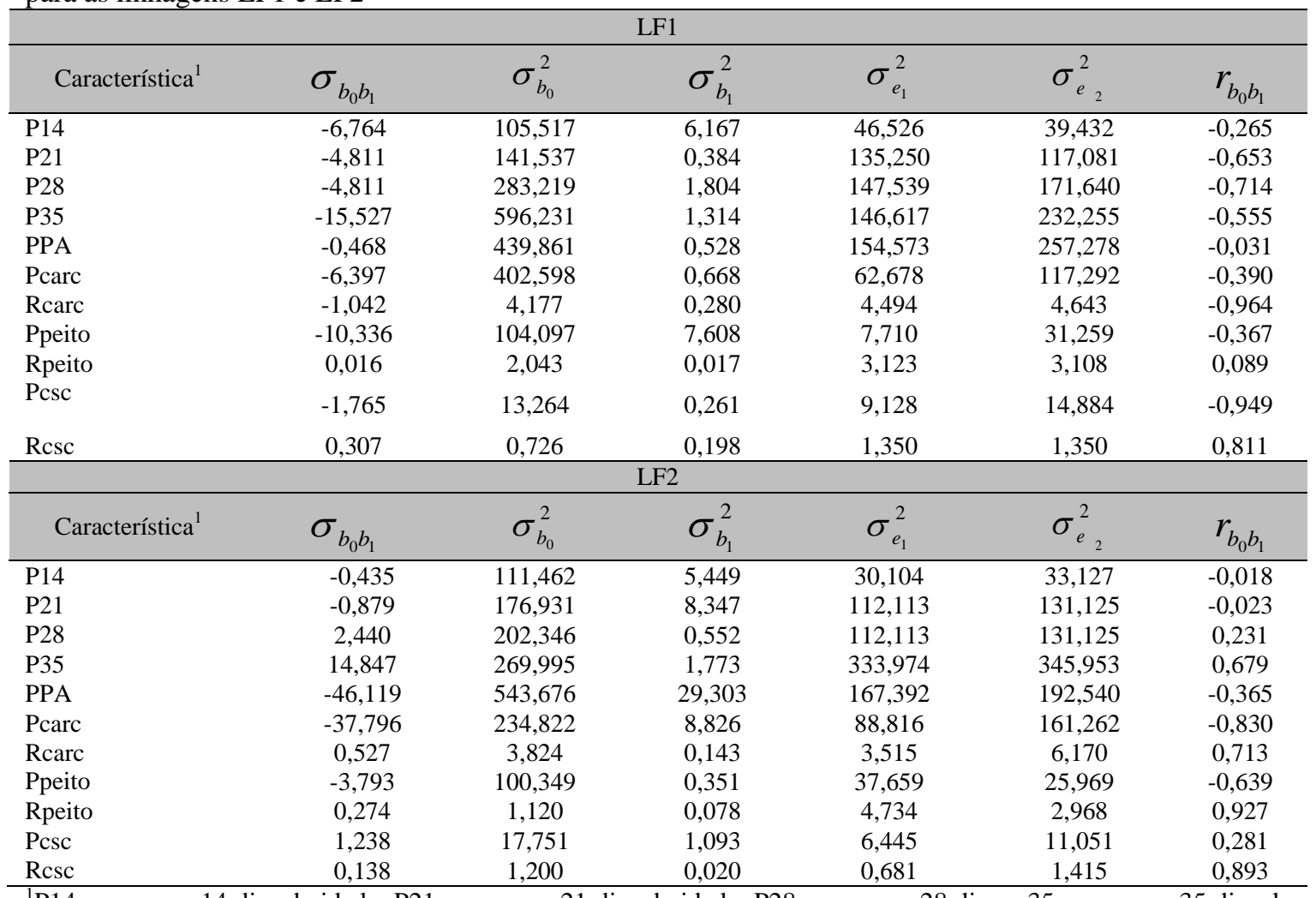

${ }^{1} \mathrm{P} 14=$ peso aos 14 dias de idade; $\mathrm{P} 21=$ peso aos 21 dias de idade; $\mathrm{P} 28=$ peso aos 28 dias; p35= peso aos 35 dias de idade; $\mathrm{PPA}=$ peso pré-abate; Pcarc = peso da carcaça; Rcarc = rendimento da carcaça; Ppeito = peso do peito; Rpeito $=$ rendimento do peito; Pcsc $=$ peso da coxa + sobrecoxa; $\operatorname{Rrcsc}=$ rendimento da coxa + sobrecoxa; $\sigma_{b_{a} b_{4}}=$ variância entre b0 e b1s $\sigma_{b_{a}}=$ variância de b0; $\sigma_{b_{1}}=$ Variância de b1; $\sigma_{c_{1}}^{2}=$ variância residual $1 ; \sigma_{\sigma_{2}}^{2}=$ variância residual $2 ; r_{b_{a} b_{1}}=$ regressão entre b0 e b1.

Houve modificações na classificação dos valores genéticos aditivos do peso corporal aos 14 dias em função dos níveis de treonina da dieta em ambas as linhagens (Fig. 1). Para peso corporal aos 21 dias de idade (LF2), ocorreram poucas modificações na dispersão dos valores genéticos aditivos.

Foi observada leve redução de dispersão dos valores genéticos em função do nível da relação treonina:lisina para peso corporal aos 21 dias de idade na linhagem LF1.
Observa-se pequena redução na dispersão dos valores genéticos do peso corporal aos 28 dias na LF1; já para a mesma idade na LF2, não ocorreu alteração na dispersão. Para peso corporal aos 35 dias na LF2, ocorreu pequeno aumento na dispersão dos valores genéticos e na LF1; para a mesma idade não foram observadas modificações na dispersão dos valores genéticos aditivos (Fig. 1). 

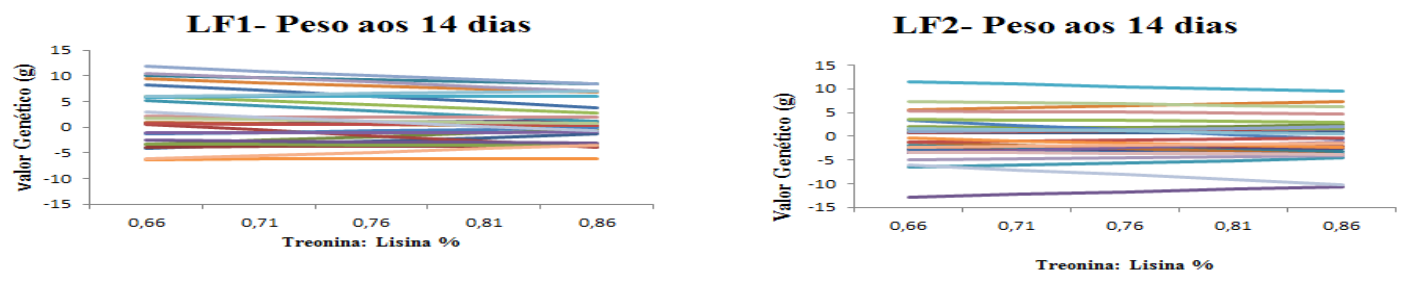

LF1- Peso aos 21 dias
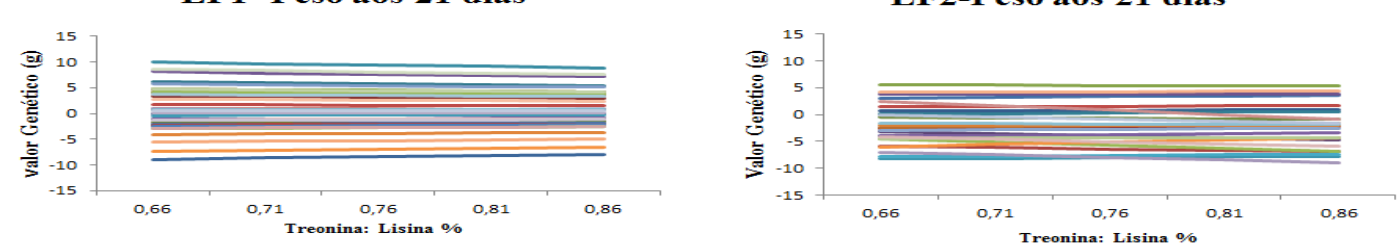

LF1- Peso aos 28 dias
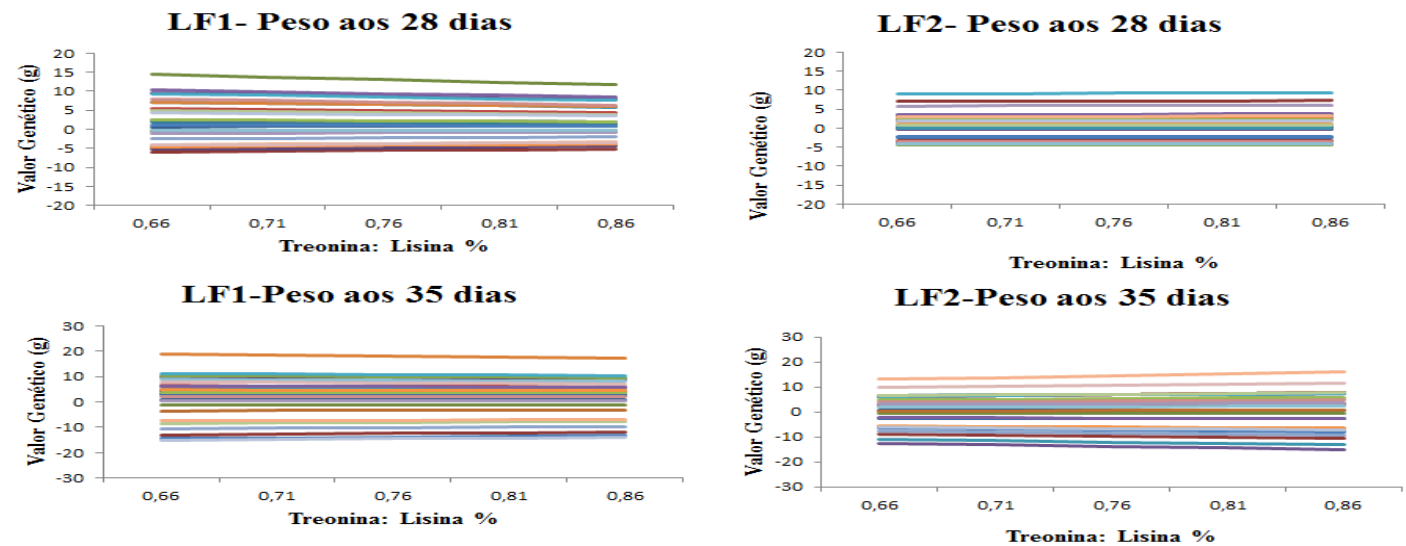

Figura 1. Normas de reação de valores genéticos aditivos de 25 indivíduos amostrados aleatoriamente para peso corporal aos 14, 21, 28 e 35 dias de idade das linhagens LF1 e LF2.

Na Fig. 2, não foram observadas modificações dos valores genéticos aditivos do peso pré-abate e peso da carcaça na linhagem LF1; não houve alteração na dispersão dos valores genéticos do peso pré-abate na linhagem LF2, mas houve alteração de posição entre os valores genéticos das codornas, o que também caracteriza interação genótipo $\mathrm{x}$ ambiente. Para peso da carcaça (LF2) e rendimento da carcaça (LF1), houve redução na dispersão dos valores genéticos; já para rendimento da carcaça (LF2), houve aumento na dispersão dos valores genéticos. Esse comportamento, em ambas as linhagens, indica sensibilidades de valores genéticos aditivos à mudança ambiental, o que caracteriza a existência de interação genótipo $\mathrm{x}$ ambiente.

Observa-se leve redução na dispersão dos valores genéticos (Fig. 3) para peso do peito (LF2) e alteração na dispersão dos valores genéticos (LF1). Observa-se aumento na dispersão dos valores genéticos para rendimento do peito (LF2); para rendimento do peito (LF1), não foi observada variação na dispersão. Houve redução dos valores genéticos do peso da coxa + sobrecoxa da linhagem LF1 e alteração da dispersão na linhagem LF2, o que caracteriza a interação genótipo $\mathrm{x}$ ambiente. Para rendimento da coxa + sobrecoxa, nas duas linhagens, ocorreu aumento da dispersão dos valores genéticos com o aumento do nível de treonina, sendo na LF1 um aumento mais intenso que na LF2. Esse comportamento indica sensibilidades de valores genéticos aditivos à mudança do gradiente ambiental, o que caracteriza a existência de interação genótipo x ambiente. Os resultados sugerem que a interação genótipo $\mathrm{x}$ ambiente interfere na expressão fenotípica de ambas as linhagens, seja pelo aumento ou redução da dispersão dos valores genéticos, seja pela alteração da classificação dos genótipos selecionados. 

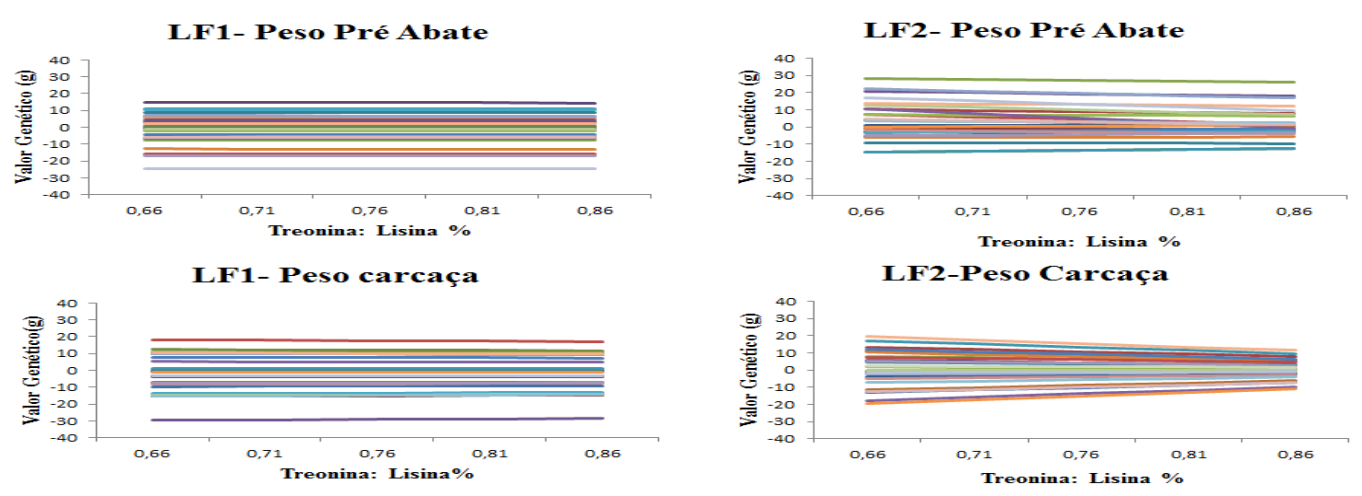

LF1 - Rendimento Carcaça

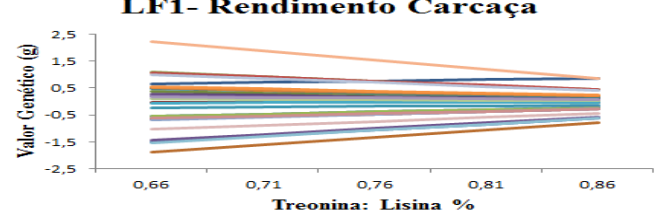

LF2- Rendimento Carcaça

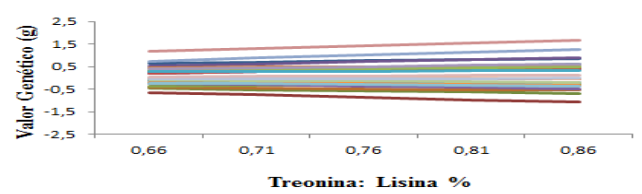

Figura 2. Normas de reação de valores genéticos aditivos de 25 indivíduos amostrados aleatoriamente para peso pré-abate, pesos e rendimentos de carcaça das linhagens LF1 e LF2.
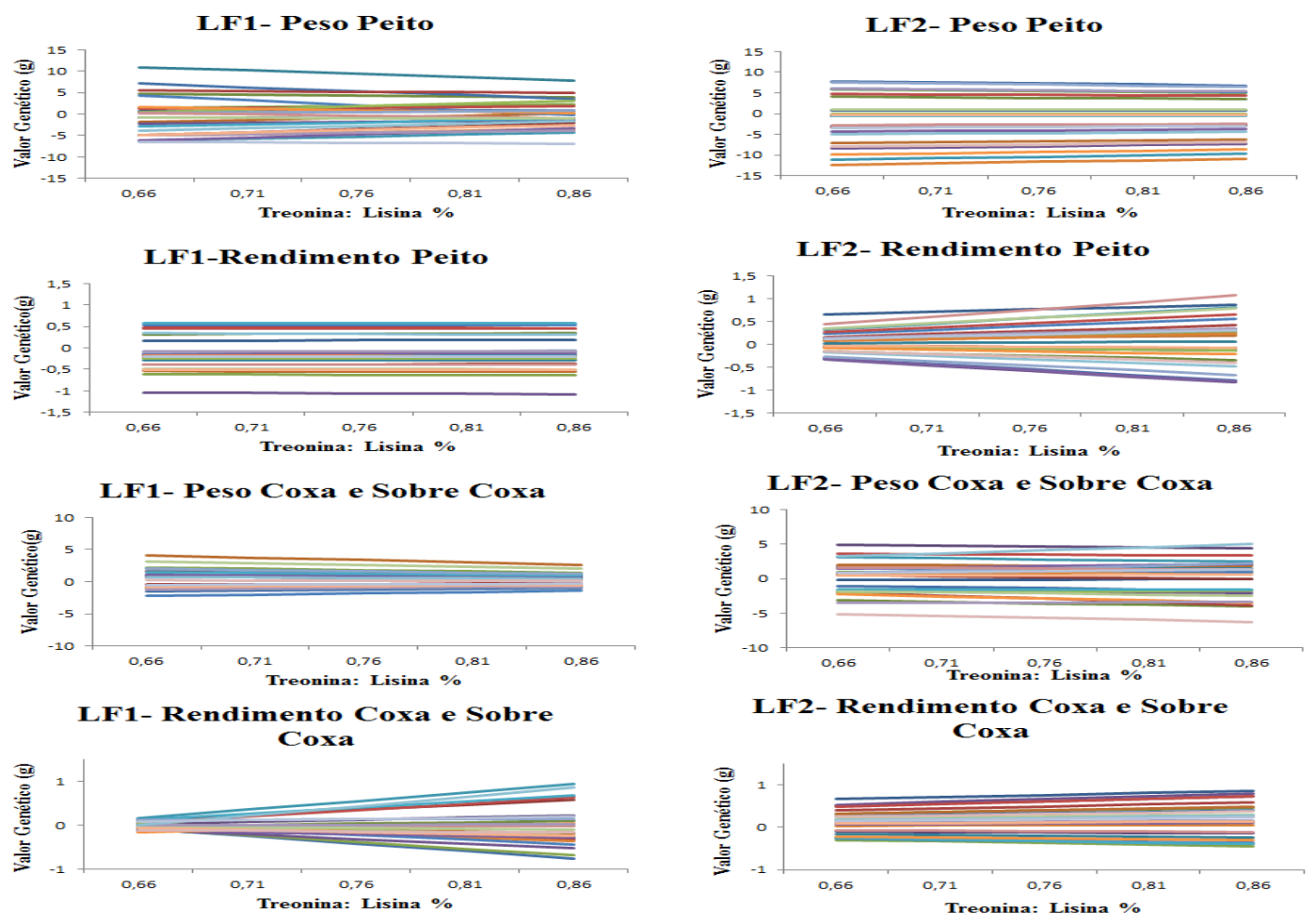

ura 3. Normas de reação de valores genéticos aditivos de 25 indivíduos amostrados aleatoriamente para pesos e rendimentos do peito e da coxa + sobrecoxa das linhagens LF1 e LF2.

As herdabilidades estimadas das características avaliadas na LF1 (Tab. 6) apresentaram maiores valores para o menor nível de treonina $(0,66)$, exceto para peso corporal aos 21 dias de idade, o que indica que ocorre crescimento da porção da variância fenotípica que é atribuída à variância genética aditiva direta em idades menores. 
Tabela 6. Estimativas de herdabilidade $\left(h^{2}\right)$ para pesos corporais de codornas de corte das linhagens LF1 e LF2, segundo as relações (treonina:lisina) da dieta

\begin{tabular}{cccccccc}
\hline \multirow{2}{*}{ Linhagem } & \multirow{2}{*}{ Característica $^{1}$} & & \multicolumn{5}{c}{ Relação treonina:lisina } \\
\cline { 3 - 7 } & & & 0,66 & 0,71 & 0,76 & 0,81 & 0,86 \\
\hline \multirow{3}{*}{ LF1 } & P21 & $h^{2}$ & 0,613 & 0,607 & 0,572 & 0,555 & 0,561 \\
& P28 & $h^{2}$ & 0,371 & 0,391 & 0,377 & 0,363 & 0,350 \\
& P35 & $h^{2}$ & 0,539 & 0,477 & 0,452 & 0,428 & 0,404 \\
& P14 & $h^{2}$ & 0,690 & 0,573 & 0,562 & 0,551 & 0,540 \\
\hline \multirow{2}{*}{ LF2 } & P21 & $h^{2}$ & 0,682 & 0,637 & 0,627 & 0,634 & 0,656 \\
& P28 & $h^{2}$ & 0,478 & 0,413 & 0,403 & 0,409 & 0,431 \\
& P35 & $h^{2}$ & 0,315 & 0,284 & 0,288 & 0,293 & 0,298 \\
\cline { 2 - 7 } & $h^{2}$ & 0,251 & 0,262 & 0,281 & 0,300 & 0,321
\end{tabular}

${ }^{\mathrm{T}} \mathrm{P} 14$ = peso aos 14 dias de idade, $\mathrm{P} 21$ = peso aos 21 dias de idade, $\mathrm{P} 28$ = peso aos 28 dias de idade, $\mathrm{P} 35=$ peso aos 35 dias de idade.

Semelhantemente ao ocorrido na LF1, as herdabilidades das características da linhagem LF2 foram, de maneira geral, maiores para o nível da relação treonina:lisina 0,66 , com exceção do peso aos 35 dias de idade. Esse comportamento indica que a seleção seria mais eficiente em níveis de treonina menores. As herdabilidades das características na linhagem LF1 (Tab. 7) para o nível de 0,66 de treonina foram maiores, exceto para as características de rendimento do peito e coxa + sobrecoxa, para as quais as herdabilidades foram maiores para $\mathrm{o}$ nível de 0,86 treonina:lisina. Na linhagem LF2, observam-se maiores herdabilidades na relação treonina:lisina de 0,66 , exceto para rendimento da carcaça, peso do peito, rendimento do peito e rendimento da coxa + sobrecoxa. Verifica-se que a seleção para a maioria das características tornase mais eficiente se praticada em níveis menores da relação treonina:lisina das dietas. As maiores estimativas da herdabilidade, observadas nas menores relação treonina:lisina das dietas, ocorreram em razão de a contribuição do efeito genético ser maior do que a dos efeitos ambientais.

Tabela 7. Estimativas de herdabilidade $\left(h^{2}\right)$ para peso pré-abate e características de carcaça de codornas de corte das linhagens LF1 e LF2, segundo as relações (treonina: lisina) da dieta

\begin{tabular}{cccccccc}
\hline \multirow{2}{*}{ Linhagem } & \multirow{2}{*}{ Característica $^{1}$} & & \multicolumn{5}{c}{ Relação treonina:lisina } \\
\cline { 3 - 7 } & PABATE & $h^{2}$ & 0,66 & 0,71 & 0,76 & 0,81 & 0,86 \\
\cline { 2 - 7 } LF1 & PCARC & $h^{2}$ & 0,773 & 0,638 & 0,632 & 0,626 & 0,620 \\
& RCARC & $h^{2}$ & 0,490 & 0,400 & 0,310 & 0,218 & 0,132 \\
& PPEITO & $h^{2}$ & 0,913 & 0,671 & 0,625 & 0,595 & 0,593 \\
& RPEITO & $h^{2}$ & 0,246 & 0,246 & 0,247 & 0,251 & 0,257 \\
& PCSC & $h^{2}$ & 0,525 & 0,357 & 0,308 & 0,259 & 0,210 \\
& RCSC & $h^{2}$ & 0,086 & 0,112 & 0,212 & 0,342 & 0,469 \\
\hline \multirow{3}{*}{ LF2 } & PABATE & $h^{2}$ & 0,703 & 0,626 & 0,585 & 0,558 & 0,551 \\
& PCARC & $h^{2}$ & 0,688 & 0,488 & 0,421 & 0,353 & 0,288 \\
& RCARC & $h^{2}$ & 0,257 & 0,196 & 0,237 & 0,282 & 0,330 \\
& PPEITO & $h^{2}$ & 0,603 & 0,674 & 0,659 & 0,644 & 0,630 \\
& RPEITO & $h^{2}$ & 0,041 & 0,106 & 0,158 & 0,218 & 0,279 \\
& PCSC & $h^{2}$ & 0,565 & 0,426 & 0,445 & 0,484 & 0,534 \\
RCSC & $h^{2}$ & 0,364 & 0,256 & 0,297 & 0,339 & 0,380 \\
\hline
\end{tabular}

${ }^{\mathrm{PABATE}}=$ peso pré- abate; $\mathrm{PCARC}$ peso da carcaça; $\mathrm{RCARC}=$ rendimento da carcaça; PPEITO = peso do peito; RPEITO = rendimento do peito; PCSC = peso da coxa+sobrecoxa; RCSC = rendimento da coxa+sobrecoxa. 


\section{CONCLUSÃO}

Modelos de regressão aleatória que consideram heterogeneidade de variância residual são melhores do que os que consideram a variância residual homogênea no estudo da sensibilidade dos valores genéticos de codornas de corte alimentadas com dieta contendo diferentes níveis da relação treonina:lisina.

Verificou-se a existência da interação genótipo $\mathrm{x}$ ambiente para as duas linhagens de codornas de corte estudadas, seja por reordenamento ou por alteração na dispersão da variância dos valores genéticos. A predição dos valores genéticos deve ser feita com o mesmo nível da relação treonina:lisina da dieta com a qual as codornas serão alimentadas no sistema de produção.

\section{AGRADECIMENTOS}

Os autores agradecem o apoio financeiro recebido do CNPQ, Capes, Fapemig e Ajinomoto.

\section{REFERÊNCIAS}

BURNHAM, K.P.; ANDERSON, D.R. Multimo del inference: understanding AIC and BIC in model selection. Sociol. Methods Res., v.33, p.261-304, 2004.

FELIPE, V.P.S.; SILVA, M.A.; WENCESLAU, R.R. et al. Utilização de modelos de norma de reação com variância residual heterogênea para estudo de valores genéticos de peso de codornas de corte em função de níveis de proteína bruta na dieta. Arq. Bras. Med. Vet. Zootec., v.64, p.991-1000, 2012.

KIRKPATRICK, M.; LOFSVOLD, D.; BULMER, M. et al. Analysis of the inheritance, selection and evolution of growth trajectories. Genetics, v.124, p.979-993, 1990
MERCADANTE, M.E.Z.; PACKER, I.U.; RAZOOK, A.G. et al. Dias ao parto de fêmea nelore de um experimento de seleção para o crescimento. IIModelo de regressão aleatória. Rev. Bras. Zootec., v.31, p.1726-1733, 2002.

MEYER, K. "WOMBAT" - Digging deep for quantitative genetic analyses by restricted maximum likelihood. In: WORLD CONGRESS ON GENETIC APPLIED TO LIVESTOCK PRODUCTION, 8., 2006, Belo Horizonte. Proceedings... Belo Horizonte: WCGALP, 2006. CD-ROM.

ROBERTSON, A. The sampling variance of the genetic correlation coefficient. Biometrics, v.15, p.469-485, 1959.

RODRIGUES, D.T. Interação genótipos ambientes em animais via modelos de normas de reação. 2012. 86f. Dissertação (Mestrado em Estatística Aplicada e Biometria). Faculdade [Ciências Agrárias], Universidade Federal de Viçosa, Viçosa, MG.

ROSTAGNO, H.S.; ALBINO, L.F.T.; DOZNELE, J.L. et al. Tabelas brasileiras para suínos e aves: composição de alimentos e exigências nutricionais. 2.ed. Viçosa: Universidade Federal de Viçosa, 2005. $186 \mathrm{p}$.

SCHWARZ, G. Estimating the dimension of a models. Ann. Stat., v.6, p.461-464, 1978.

SILVA, J.H.V.; COSTA, F.G.P. Tabela para codornas japonesas e européias. 2.ed. Jaboticabal: FUNEP, 2009. 110p.

SU, G.; MADSEN, P.; LUND, M.S. et al. Bayesian analysis of the linear reaction norm model with unknown covariates. J. Anim. Sci., v.84, p.1651-1657, 2006.

WOLFINGER, R. Covariance structures selection in general mixed models. Comun. Stat. Simul. Comput., v.22, p.1079-1106, 1993 